\title{
ANÁLISE DE POLÍTICAS PÚBLICAS DE SAÚDE: REVISÃO NARRATIVA
}

Greice Lessa1, Valdete Meurer Kuehlkamp², Alacoque Lorenzini Erdmann³, Selma Regina de Andrade ${ }^{4}$

'Enfermeira. Mestre em Saúde e Gestão do Trabalho. Universidade Federal de Santa Catarina. Orleans-SC-Brasil.

${ }^{2}$ Enfermeira. Mestre em Ciências da Saúde. Universidade Federal de Santa Catarina. Orleans-SC-Brasil.

${ }^{3}$ Enfermeira. Doutora em Enfermagem. Universidade Federal de Santa Catarina. Florianópolis-SC-Brasil.

${ }^{4}$ Enfermeira. Doutora em Enfermagem. Universidade Federal de Santa Catarina. Florianópolis-SC-Brasil.

RESUMO: Análise de políticas públicas pode ser entendida como análise das atividades realizadas por um governo com objetivo de intervir em determinada realidade ou problema social. O objetivo do estudo é realizar revisão narrativa procurando identificar os conceitos e métodos utilizados para análise de políticas. Foram adotados como descritores: Políticas de Saúde, Políticas Públicas de Saúde, Formulação de Políticas e Análise, pesquisados nas bases de dados: Literatura Internacional em Ciências da Saúde, Literatura Latino-Americana e do Caribe em Ciências da Saúde, Publisher Medline e Base de Dados de Enfermagem. A pesquisa foi realizada no período de junho de 2013 a março de 2014. Foram elegíveis 32 artigos para extração dos dados, publicados entre 2008 e 2012. Após critérios de exclusão, 05 artigos foram analisados, destes, emergiram três categorias: Bases Conceituais; Bases metodológicas e Desafios para a enfermagem. Identificou-se o Ciclo de política como o instrumento mais utilizado para analisar política.

DESCRITORES: Política de saúde; Políticas públicas de saúde; Formulação de políticas.

\section{ANALYSIS OF PUBLIC POLICIES: A NARRATIVE REVIEW}

\begin{abstract}
Analysis of public policies can be understood as the analysis of those activities undertaken by a government with the objective of intervening in a specified context or social problem. The study object is to undertake a narrative review, seeking to identify the concepts and methods used for analysis of policies. The following were adopted as descriptors: Health Policies, Public Health Policies, and Formulation of Policies and Analysis, researched in the following databases: International Literature on Health Sciences, the Latin American and Caribbean Center on Health Sciences Information, PubMed and the Base de Dados de Enfermagem (Nursing Database). The study was undertaken in June 2013 - March 2014. A total of 32 articles was eligible for the extraction of data, published between 2008 and 2012. After application of the exclusion criteria, 05 articles were analyzed, from which three categories emerged: Conceptual Bases; Methodological bases; and Challenges to nursing. The Policy cycle was identified as the instrument used most for analyzing policy. DESCRIPTORS: Health policy; Public health policies; Formulation of policies.
\end{abstract}

\section{ANÁLISIS DE POLÍTICAS PÚBLICAS DE SALUD: REVISIÓN NARRATIVA}

RESUMEN: Análisis de políticas públicas puede ser entendido como análisis de las actividades realizadas por un gobierno con objetivo de intervenir en determinada realidad o problema social. La finalidad del estudio fue realizar revisión narrativa buscando identificar los conceptos y métodos utilizados para análisis de políticas. Fueron adoptados como descriptores: Políticas de Salud, Políticas Públicas de Salud, Formulación de Políticas y Análisis, investigando en las bases de datos: Literatura Internacional en Ciencias de la Salud, Literatura Latinoamericana y de Caribe en Ciencias de la Salud, Publisher Medline y Base de Datos de Enfermería. La investigación fue realizada en el periodo de junio de 2013 a marzo de 2014. Fueron elegibles 32 artículos para obtención de los datos, publicados entre 2008 y 2012. Después de criterios de exclusión, 05 artículos fueron analizados; de estos, resultaron tres categorías: Bases conceptuales; Bases metodológicas y Desafíos para la enfermería. Se identificó el Ciclo de política como el instrumento más utilizado para analizar política.

DESCRIPTORES: Política de salud; Políticas públicas de salud; Formulación de políticas.

Universidade Federal de Santa Catarina

Rua Professor Maia, 132 - 88870-000- Orleans-SC-Brasil

E-mail: greicelessa@hotmail.com 


\section{INTRODUÇÃO}

Políticas públicas são formadas a partir de um contexto de conflitos e necessidades de diferentes segmentos da sociedade, estas são as respostas governamentais a uma diversidade de problemas sociais ${ }^{(1)}$, porém, para que estas sejam aceitas, precisam ser relevantes e possuir magnitude significativa, afim de que possam ganhar espaço na agenda de governo.

Pode-se, então, definir política pública como o campo do conhecimento que busca, ao mesmo tempo, "colocar o governo em ação" e/ou analisar essa ação (variável independente) e, quando necessário, propor mudanças no rumo ou curso dessas ações (variável dependente) $)^{(2: 26)}$.

A Análise de Políticas vai além da observação e análise dos resultados da política, ela se preocupa com o processo de construção da política pública, focando na definição da agenda e destacando aspectos como, por exemplo, os interesses dos atores que participam do jogo político, a interação entre eles, a arquitetura de poder e tomada de decisões, conflitos e negociações, etc ${ }^{(3)}$. "Refere-se ao estudo das ações realizadas, ou não, pelo governo, na tentativa de alcançar objetivos em relação a um problema ou conflito social" (4:512).

As não ações também devem ser analisadas e discutidas, pois estas são consideradas uma forma de manifestação política e traduzem a opção política dos decisores ${ }^{(5)}$. Para analisar políticas faz-se necessário a existência de modelos teóricos que possibilitem a compreensão da realidade, os sentidos e os significados dos processos envolvidos nas operações ${ }^{(4)}$.

Existem diferentes modelos de que permitem analisar políticas públicas como, por exemplo, a classificação em tipos de políticas (distributivas, redistributivas, regulatórias e constitutivas); o incrementalismo; o modelo denominado "lata de lixo"; o modelo dos múltiplos fluxos; o modelo das coalisões de defesa; as arenas sociais; o modelo do equilíbrio interrompido; o ciclo da política pública (policycycle) entre outros ${ }^{(5)}$.

Neste contexto, a análise de políticas de saúde vem se consolidando no Brasil com um conjunto de diferentes abordagens metodológicas. Este artigo se propõe a identificar, com base na literatura, os principais conceitos e métodos utilizados para análise de políticas, focalizando as políticas públicas de saúde, com vistas à ampliação do conhecimento e aplicabilidade na enfermagem.

\section{MÉTODO}

Trata-se de revisão narrativa ${ }^{(6)}$ a respeito do tema, procurando identificar por meio da literatura produzida sobre Análise de Política. A revisão narrativa permite a descrição e discussão teórico-reflexiva de estudos já produzidos a respeito de um assunto relevante e atual, de modo que ainda não há possibilidade de realização de uma análise sistematizada. Também, viabiliza o acesso bem como o processamento do conhecimento e de ideias novas acerca da temática estudada, possibilitando a sua emolduração de forma sumarizada e em espaço temporal curto $^{(7)}$.

A pesquisa abrangeu artigos publicados acerca da análise de política no período recente (2008 a 2012). Foram adotados como descritores: Políticas de Saúde, Políticas Públicas de Saúde, Formulação de Políticas e Análise, nos idiomas inglês, português e espanhol, sendo pesquisados nas bases de dados Literatura Internacional em Ciências da Saúde (Medline), Literatura LatinoAmericana e do Caribe em Ciências da Saúde (LILACS), e Base de Dados de Enfermagem (BDENF). Para a base dados do Publisher Medline (Pubmed) foram utilizados os mesmos descritores traduzidos para o inglês.

Foram excluídos os trabalhos que abordavam: Editoriais; Cartas; Artigos de opinião; Comentários; Resumos de Anais; Ensaios; Publicações duplicadas; Boletins epidemiológicos; Relatórios de Gestão; Materiais publicados em outro idioma que não fosse espanhol, inglês e português; e Estudos que não contemplassem o escopo desta pesquisa.

Em artigos localizados por mais de uma estratégia de busca foi realizada a supressão dos repetidos. Durante a análise dos trabalhos iniciouse a leitura dos títulos, seguida da leitura dos resumos e, posteriormente, dos textos completos. A aplicação dos critérios de exclusão foi realizada em todas as etapas, sempre por consenso de dois leitores. 
Os artigos selecionados foram analisados por meio de síntese qualitativa a partir do guia de orientações ${ }^{(7-8)}$ de forma a caracterizar o estudo realizado: Tipo de política: suas bases conceituais e estratégias metodológicas utilizadas para análise de políticas e a aplicabilidade para as práticas da enfermagem. Ainda é escassa a literatura acerca da temática estudada, o que impossibilita de tal forma uma análise mais criteriosa como seria a exemplo de uma revisão integrativa ou sistemática. Dessa forma, a revisão narrativa se mostrou como alternativa mais adequada.

\section{RESULTADOS}

A busca realizada localizou 474 artigos, dos quais 32 foram selecionados para a extração dos dados. Aplicando-se os critérios de exclusão eliminou-se 27, sendo a pesquisa realizada com 05 artigos (Figura 1).

No Quadro 1 estão apresentados os dados referentes à autoria, data, ano, local, período de publicação, bem como: tipo de política, base conceitual, método utilizado e estratégias metodológicas.

\section{Quais os conceitos e ferramentas frequentemente utilizados Análise de Políticas?}

Descritores: Políticas de Saúde,
Políticas Públicas de Saúde,
Formulação de Políticas, Análise. Seleção: 473 artigos

\begin{tabular}{|c|c|c|c|}
\hline $\begin{array}{l}441 \text { artigos removidos por } \\
\text { duplicata e critérios de exclusão }\end{array}$ & \begin{tabular}{|l|}
32 Artigos \\
potenciais
\end{tabular} & $\begin{array}{l}27 \text { artigos } \\
\text { excluídos }\end{array}$ & $\begin{array}{l}5 \text { artigos incluídos } \\
\text { na revisão }\end{array}$ \\
\hline
\end{tabular}

Figura 1 - Fluxograma de seleção dos trabalhos identificados. Florianópolis-SC, 2014

Quadro 1 - Descrição dos estudos sobre Análise de Política. Florianópolis-SC, 2014

\begin{tabular}{|c|c|c|c|c|}
\hline $\begin{array}{l}\text { Autor, Data, } \\
\text { Ano, Local, } \\
\text { Período } \\
\end{array}$ & $\begin{array}{l}\text { Tipo de } \\
\text { Política }\end{array}$ & Base Conceitual & $\begin{array}{l}\text { Método } \\
\text { Utilizado }\end{array}$ & $\begin{array}{c}\text { Estratégias } \\
\text { Metodológicas }\end{array}$ \\
\hline $\begin{array}{l}\text { Machado; } \\
\text { Salvador; } \\
\text { O'Dwyer, } \\
2011^{(9)} \\
2003 \text { e } 2010 . \\
\text { Brasil }\end{array}$ & $\begin{array}{l}\text { Serviço de } \\
\text { Atendimento } \\
\text { Móvel de } \\
\text { Urgência }\end{array}$ & & $\begin{array}{l}\text { Análise de } \\
\text { políticas } \\
\text { públicas. }\end{array}$ & $\begin{array}{l}\text { Revisão } \\
\text { bibliográfica, análise } \\
\text { de documentos, } \\
\text { dados oficiais e } \\
\text { entrevistas. }\end{array}$ \\
\hline $\begin{array}{l}\text { Pinto; } \\
\text { Teixeira, } \\
2011^{(10)} \\
2007-2008 \\
\text { Brasil } \\
\end{array}$ & $\begin{array}{l}\text { Política de } \\
\text { Gestão do } \\
\text { Trabalho e } \\
\text { Educação } \\
\text { na Saúde }\end{array}$ & $\begin{array}{l}\text { Análise de política compreende analisar os cincos } \\
\text { passos que compõem a política: determinação } \\
\text { da agenda, formulação e legitimação da política, } \\
\text { implementação das políticas e avaliação de políticas. }\end{array}$ & $\begin{array}{l}\text { Teoria do } \\
\text { ciclo da } \\
\text { política de } \\
\text { Kingdon }\end{array}$ & $\begin{array}{l}\text { Análise documental } \\
\text { e observação } \\
\text { participante. }\end{array}$ \\
\hline $\begin{array}{l}\text { Trevisani; } \\
\text { Burlandy; } \\
\text { Constante, } \\
2012^{(11)} \\
2008 \text { - } 2009 \\
\text { Brasil }\end{array}$ & $\begin{array}{l}\text { Politicas de } \\
\text { saúde do } \\
\text { Programa } \\
\text { Bolsa } \\
\text { Família }\end{array}$ & $\begin{array}{l}\text { A análise de política visa explicar a repercussão } \\
\text { gerada pelos mecanismos e efeitos da formulação } \\
\text { da política sobre as decisões e processos na } \\
\text { própria criação da política, interferindo na } \\
\text { escolha de determinados conteúdos políticos. }\end{array}$ & $\begin{array}{l}\text { Análise de } \\
\text { múltiplos } \\
\text { fluxos, } \\
\text { proposto } \\
\text { por } \\
\text { Kingdon. }\end{array}$ & $\begin{array}{l}\text { Análise da legislação } \\
\text { e de documentos } \\
\text { oficiais e entrevistas. }\end{array}$ \\
\hline $\begin{array}{l}\text { Leal, } \\
\text { Figueiredo, } \\
\text { Nogueira-da- } \\
\text { Silva, 2012 } \\
\text { 2010-2012 } \\
\text { Brasil }\end{array}$ & $\begin{array}{l}\text { Política } \\
\text { Nacional de } \\
\text { Atenção } \\
\text { Integral à } \\
\text { Saúde do } \\
\text { Homem }\end{array}$ & $\begin{array}{l}\text { Análise de Política não se trata de uma simples } \\
\text { mensuração da distância entre os objetivos } \\
\text { e diretrizes enunciados nos documentos da } \\
\text { Política. As políticas devem ser compreendidas } \\
\text { no contexto de sua execução e que a } \\
\text { implementação da política pública diz respeito } \\
\text { às pessoas que de fato a implementam. }\end{array}$ & $\begin{array}{l}\text { Referencial } \\
\text { da } \\
\text { Antropologia, } \\
\text { com } \\
\text { técnica da } \\
\text { etnografia. }\end{array}$ & $\begin{array}{l}\text { Análise documental, } \\
\text { entrevista e } \\
\text { observação. }\end{array}$ \\
\hline $\begin{array}{l}\text { Rendeiro, } \\
2011^{(13)} \\
2000-2010 \\
\text { Brasil }\end{array}$ & $\begin{array}{l}\text { Política de } \\
\text { Saúde Bucal }\end{array}$ & $\begin{array}{l}\text { Análise de política deve obedecer a rigorosos } \\
\text { procedimentos metodológicos e científicos, que } \\
\text { possibilitem ao mesmo tempo avançar na produção } \\
\text { do conhecimento e orientar as ações deste processo } \\
\text { complexo que é a tomada de decisões do Estado, } \\
\text { diante de uma série de questões e possibilidades. }\end{array}$ & $\begin{array}{l}\text { Ciclo de } \\
\text { Política. }\end{array}$ & $\begin{array}{l}\text { Documental, } \\
\text { entrevista. }\end{array}$ \\
\hline
\end{tabular}


Os estudos selecionados foram publicados no período de 2011 a 2012, sendo a coleta de dados realizada entre 2000 e 2012. Em relação à política analisada identificou-se o Serviço de Atendimento Móvel de Urgência, a Política de Gestão do Trabalho e Educação na Saúde, a Política de Saúde do Programa Bolsa Família, a Política Nacional de Atenção Integral à Saúde do Homem e a Política de Saúde Bucal. Na análise de dados emergiram três categorias: Bases Conceituais, Bases Metodológicas e Desafios para a Enfermagem.

Dos cinco artigos selecionados quatro apresentavam base conceitual sobre análise de política. O método utilizado para análise da política compreendeu, na maioria dos artigos. O ciclo de política, identificando-se também a análise de múltiplos fluxos e o referencial da antropologia. Como método de coleta de dados os autores utilizaram: revisão bibliográfica, análise documental, entrevista e observação participante. Não foi identificada a aplicabilidade da análise de política para a enfermagem nos artigos selecionados.

\section{DISCUSSÃO}

Nas últimas décadas as políticas públicas têm se tornado um campo de pesquisa cada vez mais explorado e atraem a atenção de diversos grupos, dentre os quais gestores e pesquisadores ${ }^{(3,14)}$. E um campo de pesquisa, relativamente, consolidado no exterior e, cada vez mais, também no Brasil( ${ }^{(3)}$.

Prova disso é o fato de o Ministério da Saúde (MS) estar interessado em patrocinar um número cada vez mais elevado de publicações voltadas principalmente para estudos e análises de políticas. Este fato garante legitimidade ao campo e contribui para a consolidação e profissionalização da análise de políticas no Brasil $^{(15)}$.

Alguns fatores contribuíram para a crescente visibilidade desta área, como: a adoção de políticas restritivas de gasto, novas visões sobre o papel dos governos que substituíram as políticas keynesianas do pós-guerra por políticas restritivas de gasto, além do fato de os países em desenvolvimento ainda não serem capazes de formular políticas que impulsione o desenvolvimento econômico e promova a inclusão social de grande parte de sua população ${ }^{(3)}$.
A política pública enquanto área de conhecimento e disciplina acadêmica surgiu nos EUA com ênfase nos estudos sobre a ação dos governos. Estes estudos levam em consideração o fato de que em democracias estáveis, as ações realizadas ou não pelo governo são passíveis de ser formuladas cientificamente e analisadas por pesquisadores independentes ${ }^{(3)}$.

Apesar do crescente interesse nas últimas décadas na análise de política, observou-se neste estudo que as publicações de artigos relacionados ao tema ainda é incipiente. Os cinco artigos selecionados foram publicados a partir de 2011, o que identifica a não publicação de artigos com essa temática no período de 2008 a 2010. Dessa forma, a discussão segue em três categorias: Bases Conceituais, Bases Metodológicas e Desafios para Enfermagem.

\section{Análise de Políticas: Bases Conceituais}

Na Análise de Política a palavra política é diferenciada em três dimensões: a "polity", que se refere às instituições políticas e ao sistema político, "politics" na dimensão processual, ou seja, o processo político e "policy" que se refere às políticas públicas em $\mathrm{si}^{(16)}$. Os artigos selecionados ${ }^{(10-11)}$ trouxeram como base conceitual a análise dos passos que compõem a política: determinação da agenda, formulação e legitimação da política, implementação e avaliação de políticas, compreendendo seus mecanismos e efeitos, visando explicar como esses repercutem sobre decisões e processos, interferindo em escolhas de determinados conteúdos de políticas, em detrimento de outros.

Outro ponto importante identificado nos artigos selecionados ${ }^{(13-14)}$ é que as políticas devem ser compreendidas no contexto de sua execução e que a sua implementação diz respeito às pessoas que de fato a implementam. Ela deve obedecer a rigorosos procedimentos metodológicos e científicos, avançar na produção do conhecimento e orientar as ações para a tomada de decisões do Estado.

É necessário destacar a diferença entre Análise de Política e Avaliação de Política. A avaliação se fundamenta na apreciação dos resultados da política, atentando para categorias como eficiência, eficácia e efetividade, derivados da 
comparação entre metas e resultados ${ }^{(17)}$. Já a Análise se preocupa com o processo de construção da política pública, principalmente, no que se refere à definição da agenda governamental ${ }^{(3)}$.

\section{Análise de Políticas: Bases Metodológicas}

Para analisar mais profundamente o surgimento e o percurso de um programa político, deve avaliar intensamente o ciclo da política, tornando necessário conhecer os arranjos institucionais, as atitudes e objetivos dos atores políticos, os instrumentos de ação e as estratégias políticas ${ }^{(16: 226)}$.

Para tanto, o ciclo de política divide-se em cinco momentos sucessivos, interligados e dinâmicos: identificação de problemas, conformação da agenda, formulação, implementação e avaliação da política ${ }^{(3)}$.

Neste estudo identificou-se que o método mais utilizado para análise de política foi o ciclo de política ${ }^{(10-11,13)}$ envolvendo os cinco passos citados anteriormente. Observou-se também que dois artigos utilizaram o ciclo de política na abordagem de Kingdon ${ }^{(10-11)}$. Este autor propõe que o processo de tomada de decisão nas políticas públicas poderia ser representado pela ligação de três grandes correntes dinâmicas: a corrente dos problemas, a propostas ou alternativas e a corrente da política. Neste sentido, a agenda governamental segue um processo não intencional que se caracteriza por: surgimento ou reconhecimento de um problema pela sociedade em geral; existência de ideias e alternativas para conceituá-los e contexto político, administrativo e legislativo favorável ao desenvolvimento da ação ${ }^{(18)}$.

\section{Análise de Políticas: Desafios para a Enfermagem}

Para a enfermagem, conhecer as políticas públicas de saúde é de primordial importância, uma vez que toda a implementação das ações relacionadas ao cuidado individual e coletivo parte da proposta política estabelecida.

$\mathrm{Na}$ atualidade essas políticas estão sendo implementadas de acordo com o modelo médico vigente por meio de "pacotes", que contemplam as necessidades da população de modo único, descaracterizando, assim, as peculiaridades de cada realidade da população ${ }^{(19)}$.

É fundamental ressaltar que, as intervenções de enfermagem devem se dar por meio de três dimensões: a estrutural, que corresponde ao aparato jurídico-político e ideológico, além da infraestrutura econômica; a particular - que se refere aos processos relativos aos grupos sociais; a singular, que se relaciona à expressão biopsíquica no corpo individual e social ${ }^{(20)}$.

O comprometimento da enfermagem com as políticas públicas de saúde inicia no processo de formação do enfermeiro, aonde as ações de promoção da saúde bem como de prevenção dos agravos foram aos poucos sendo inclusos no processo de educação e culminaram em mudança curricular, corroborando com a nova visão e mudança de paradigma em construção na área da saúde nacional.

Nesse cenário, mudanças conceituais na forma de pensar e fazer saúde precisam estar articulados com todo o contexto social e ambiental ${ }^{(21)}$. Este processo não requer mudanças isoladas e sim a articulação entre ensino-aprendizagem na formação do enfermeiro, envolvendo desafios na busca para a melhoria da atenção básica, além de englobar as práticas de promoção à saúde, questões éticas e políticas articulando processo ensino-serviço ${ }^{(22)}$.

Um exemplo é o Programa Saúde da Família (PSF), hoje Estratégia Saúde da Família (ESF), que surgiu como forma de responder efetivamente aos princípios do Sistema Único de Saúde (SUS). Os profissionais envolvidos tanto na construção como na consolidação do sistema devem possuir previamente, habilidades para atuar na promoção da saúde, por meio do foco principal que é a prevenção de agravos à saúde da população e que respondam de forma satisfatória e adequada às problemáticas sociais e de saúde de toda uma população que recebe atendimento nos serviços de saúde ${ }^{(23)}$.

É muito comum à enfermagem atuar na gestão dos serviços de saúde, e a estruturação, elaboração e o lançamento de uma política necessita diretamente do acompanhamento do processo de implementação por parte dos gestores dessas políticas ${ }^{(24)}$.

Em um estudo desenvolvido sobre as políticas e tecnologias de gestão, em serviços de saúde e de enfermagem, identificou-se que o SUS 
foi tratado como centralidade em todos os contextos discutidos, com ênfase ao princípio da integralidade e na descentralização da atenção à saúde. Estes dois princípios requerem que o enfermeiro esteja envolvido, se instrumentalize e esteja preparado e comprometido, a fim de que desenvolva as suas diferentes habilidades, capacidades e competências, não apenas técnicas, mas também políticas, tecnológicas e de gestão ${ }^{(25)}$.

De fato, todas as etapas que dizem respeito ao processo de trabalho sendo elas: administrar, organizar, coordenar, acompanhar, tomar decisões e avaliar as ações já fazem parte da rotina de trabalho do enfermeiro. É necessário que esse profissional se apodere de conhecimentos acerca das políticas públicas, suas diretrizes, no direcionamento e implementação das ações. Incumbe-Ihe ainda participar, opinar, decidir e intervir quando necessário uma vez que a dimensão política se concretiza a partir da capacidade de união das pessoas em grupos sociais, com necessidades e interesses representativos da equipe e dos pacientes de acordo com a demanda em questão(26).

Nesse sentido, a compreensão pela enfermagem da forma como se estruturam as políticas sociais, entre elas as políticas de saúde, é fundamental para que as intervenções tanto na promoção da saúde quanto na prevenção dos agravos, contemplem a realidade das problemáticas vivenciadas pela população, respondendo de forma resolutiva.

\section{CONCLUSÕES}

As produções sobre Análise de Políticas ainda são incipientes no Brasil, uma vez que poucos estudos são realizados com base nesta temática, porém, há um aumento de publicações na área, sobretudo a partir de 2011. Os estudos desenvolvidos utilizam em sua maioria o método do ciclo de política como instrumento para analisar a política.

Sugere-se então, a realização e publicação de novos estudos, com maior rigor metodológico, buscando ampliar a gama de conhecimentos que fundamentem a análise de políticas, auxiliando na sua compreensão e tomada de decisão.

Há também a necessidade de discutir a análise de política com foco na enfermagem, uma vez que estes profissionais estão diretamente relacionados à aplicabilidade dessas políticas.

Neste estudo foram descritos e discutidos aspectos relacionados à análise de políticas de saúde e podem ter ocorrido vieses relacionados à avaliação crítica das autoras, uma vez que se utilizou literatura com apreciação crítica pessoal dos autores.

\section{REFERÊNCIAS}

1. Viana AL. Enfoques metodológicos em políticas públicas: novos referenciais para estudos sobre políticas sociais. In: Canesqui AM, organizador. Ciências sociais e saúde. São Paulo: Hucitec; 1997.

2. Souza C. Políticas públicas: uma revisão da literatura. Sociologias. [Internet] 2006;16(8) [acesso em 04 jul 2013]. Disponível em: http://dx.doi.org/10.1590/S151745222006000200003

3. Serafim M, Dias RB. Análise de política: uma revisão da literatura. Cadernos Gestão Social. 2012;3(1):121-34.

4. Gottems BD, Pires MRGM, Calmon PCDP, Alves ED. O modelo dos múltiplos fluxos de Kingdon na análise de políticas de saúde: aplicabilidades, contribuições e limites. Saúde Soc.[Internet] 2013;22(2) [acesso em 09 nov 2013]. Disponível em: http://dx.doi.org/10.1590/ S0104-12902013000200020

5. Machado SHS. O uso da teoria de stakeholders em uma análise da etapa de formulação da política nacional de medicamentos. Rev. Adm. Pública [Internet] 2013;47(3) [acesso em 10 nov 2013]. Disponível em: http://dx.doi. org/10.1590/S0034-76122013000300001

6. Rother E T. Revisão sistemática $x$ revisão narrativa. Acta Paul. Enferm. [Internet] 2007;20(2) [acesso em 20 jan 2014]. Disponível em: http://dx.doi.org/10.1590/ S0103-21002007000200001

7. Rodgers M, Arai L, Britten N, Petticrew M, Popay J, Roberts $\mathrm{H}$, et al. Narrative synthesis in systematic reviews. Manchester: ESRC Research Methods Programme; 2007.

8 Aveyard H. Doing a literature review in health and social care: a practical guide. Maidenhead: Open University Press; 2007.

9. Machado CV, Salvador FGF, O'Dwyer G. Serviço de atendimento móvel de urgência: análise da política brasileira. Rev. Saúde Pública. [Internet] 2011;45(3) [acesso em 01 ago 2013]. Disponível em: http://www. scielo.br/scielo.php?script=sci_arttext\&pid=S003489102011000300010\&lng=pt\&nrm=iso\&tlng=en 
10. Pinto ICM, Teixeira CF. Formulação da política de gestão do trabalho e educação na saúde: o caso da Secretaria Estadual de Saúde da Bahia, Brasil, 20072008. Cad. Saúde Pública. 2011;27(9):1777-99.

11. Trevisani JJD, Burlandy L, Constante JP. Fluxos decisórios na formulação das condicionalidades de saúde do programa bolsa família. Saúde Soc. 2012; 21(2): 492-509.

12. Leal AF, Figueiredo WS, Nogueira-da-Silva GS, Charting the Brazilian Comprehensive Healthcare Policy for Men (PNAISH), from its formulation through to its implementation in local public health services. Ciênc. Saúde Colet. 2012;17(10):2607-16.

13. Rendeiro MMP. O Ciclo da política de saúde bucal no sistema de saúde Brasileiro: atores, ideias e Instituições [tese]. Rio de Janeiro (RJ): Escola Nacional de Saúde Pública Sergio Arouca; 2011.

14. Cortes SV, Lima LL. A contribuição da sociologia para a análise de políticas públicas. Lua Nova. 2012;87:3362.

15. Vaitsman JR, Mendes J, Lobato LVC. Análise de políticas, políticas de saúde e a Saúde Coletiva. Physis. 2011; 23 (2):589-611.

16. Frey K. Políticas públicas: um debate conceitual e reflexões referentes à prática da análise de políticas públicas no Brasil. Planejamento e Políticas Públicas. 2000;21:211-59.

17. Cavalcanti PA. O conceito de avaliação de políticas, programas e projetos. In: Perez JRR. Estudo, pensamento e criação: planejamento educacional e avaliação na escola. Campinas: FE/Unicamp; 2005.

18. Kingdon JW. Agendas, alternatives and public policies. 2. ed. Ann Arbor: University of Michigan; 2003.

19. Corrêa ACP, Ferriani MGC . A produção científica da enfermagem e as políticas de proteção à adolescência. Rev. bras. enferm. [Internet] 2005;58(4) [acesso em 10 jan 2014]. Disponível: http://dx.doi.org/10.1590/ S0034-71672005000400013

20. Bertolozzi M.R, Greco R.M. As Políticas de saúde do Brasil: reconstrução histórica e perspectivas atuais. Rev. Esc. Enferm USP. [Internet] 1996;30(3) [acesso em 10 jan 2014]. Disponível: http://www.ee.usp.br/ reeusp/upload/pdf/356.pdf

21. Costa KSC, Miranda FAN. Sistema Único de Saúde na formação acadêmica do enfermeiro. Rev. bras. enferm. [Internet] 2009;62(2) [acesso em 10 jan 2014]. Disponível: http://dx.doi.org/10.1590/S003471672009000200021

22. Silva kL, Sena RR, Grillo MJC, Horta NC, Prado $P M C$. Educação em enfermagem e os desafios para a promoção de saúde. Rev. bras. enferm. 2009; 62(1):8691.

23. Santos BRL, Moraes EP, Piccinini GC, Sagebin HV, Eidt OR, Witt RR. Formando o enfermeiro para o cuidado à saúde da família: um olhar sobre o ensino de graduação. Rev bras enferm 2000; 53(esp):49-59.

24. Willig $\mathrm{MH}$, Lenardt $\mathrm{MH}$, Méier MJ. A trajetória das políticas públicas do idoso no Brasil: breve análise. Cogitare enferm. 2012;17(3):574-7.

25. Lopes MMB, Carvalho JN, Backes MTS, Erdmann AL, Meirelles BHS. Políticas e tecnologias de gestão em serviços de saúde e de enfermagem. Acta Paul Enferm. 2009;22(6): 819-27.

26. Persegona KR, Rocha DLB, Lenardt MH, Zagonel IPS. O conhecimento político na atuação do enfermeiro. Esc Anna Nery. 2009;13(3): 645-50. 